\title{
Diagnóstico prenatal de anomalías cromosómicas
}

\section{Prenatal diagnosis of chromosomal abnormalities}

\author{
Graciela Alegre ${ }^{1}$, Camila Anderlini ${ }^{2}$, María Victoria Sadir $^{2}$, Stehli Carolina Anita ${ }^{2}$, Cuestas Eduardo ${ }^{3}$ \\ 1Universidad Católica de Córdoba. Facultad de Ciencias de la Salud. Cátedra de Inmunología. Clínica Universitaria Reina Fabiola, Servicio de Alergia e \\ Inmunología. \\ 2Universidad Católica de Córdoba. Facultad de Ciencias de la Salud. Estudiantes de quinto año, Carrera de Medicina. \\ 3Universidad Católica de Córdoba. Facultad de Ciencias de la Salud. Catedra de Bioestadística \\ Correspondencia: Graciela Alegre. Servicio de Alergia e Inmunología- Clínica Universitaria Reina Fabiola. Oncativo 1248 -X5004FHP- Córdoba, Argentina. e- \\ mail: graalegre98@gmail.com.
}

\section{Resumen}

INTRODUCCION: Las anomalías cromosómicas son alteraciones en los cromosomas sexuales o de los autosomas que pueden ser estructurales o numéricas. El Síndrome de Down o trisomía 21 es una de las anomalías cromosómicas más frecuentes, donde el 95\% de las afecciones se deben a una trisomía completa. Estas alteraciones generan un importante aumento de la morbimortalidad, por lo cual es importante conocer factores de riesgo y sospecharlas prenatalmente. Los métodos más utilizados para el screening prenatal de estas anomalías son los marcadores serológicos y las pruebas de imágenes ecográficas. Otras pruebas de diagnóstico prenatal no invasivas que se pueden utilizar se basan en la detección ADN fetal que circula libremente en plasma materno.

METODOLOGÍA: A partir de un escenario clínico se planteó una pregunta clínica estructurada. Se realizó una búsqueda bibliográfica en Pubmed con el objetivo de encontrar un artículo que pueda responder a nuestro interrogante sobre si el estudio del ADN fetal circulante en comparación con el screening combinado es un método diagnóstico más sensible y específico para puede detectar el Síndrome de Down. Se seleccionó el siguiente artículo: "Cell-free DNA Analysis for Noninvasive Examination of Trisomy". ANALISIS DEL ARTICULO: Para poder responder a nuestra pregunta clínica estructurada, se realizó un análisis secundario de los datos de las distintas variables calculando los valores de sensibilidad (S), especificidad (E), valor predictivo positivo (VPP), valor predictivo negativo (VPN) y su respectivo intervalo de confianza del 95\% (IC 95\%). Destacamos también el resultado del área bajo la curva (ABC) de la curva ROC (Receiver Operating Characteristic).

CONCLUSIÓN: el screening con ADN fetal presenta una mayor sensibilidad y especificidad frente al screening combinado (ecografía y pruebas bioquímicas) para el diagnóstico del Síndrome de Down. Pero su elevado costo implica aún un limitante a resolver.

Palabras claves: diagnóstico prenatal, síndrome de down, anomalía cromosómica.

\section{Abstract}

INTRODUCTION: Chromosomal abnormalities are alterations in sex or autosomes chromosomes, they can be structural or numerical. Down syndrome or trisomy 21 is one of the most frequent chromosomal abnormalities, where $95 \%$ of the conditions are due to a complete trisomy. These alterations produces a significant increase in morbidity and mortality, it is important to know risk factors and suspect them

Revista Methodo: Investigación Aplicada a las Ciencias Biológicas. Facultad de Medicina. Universidad Católica de Córdoba. Jacinto Ríos 571 Bo Gral. Paz. X5004FXS. Córdoba. Argentina. Tel.: (54) 3514517299 / Correo: methodo@ucc.edu.ar / Web: methodo.ucc.edu.ar| BIOESTADISTICA Y METODOLOGIA APLICADA Methodo 2019;4 (4):148-152 
prenatally. The most used methods for prenatal screening of these abnormalities are serological markers and ultrasound imaging tests. The detection of fetal DNA that circulates freely in maternal plasma is an other non-invasive prenatal diagnostic tests that can be used.

METHODOLOGY: A structured clinical question was raised from a clinical setting. A bibliographic search was conducted in Pubmed in order to finding an article that could answer our structured question about if the study of circulating fetal DNA compared to combined screening is a more sensitive and specific diagnostic method to detect Down syndrome. The article whose title is: "Cell-free DNA Analysis for Noninvasive Examination of Trisomy", was selected.Analysis of the article: In order to answer our structured clinical question, a secondary analysis was performed to calculate sensitivity (S), specificity (E), positive predictive value (VPP), negative predictive value ( NPV) and its respective 95\% confidence interval $(95 \% \mathrm{CI})$. We also highlight the result of the area under the curve (ABC) of the ROC (Receiver Operating Characteristic) curve.

CONCLUSION: fetal DNA screening has higher sensitivity and specificity compared to combined screening (ultrasound and biochemical tests) for the diagnosis of Down syndrome. But its high cost still implies a limitation to solve.

Keywords: prenatal diagnosis, down syndrome, chromosomal abnormality.

\section{Escenario clínico}

Paciente femenina, previamente sana, de 36 años cursando el primer trimestre de embarazo (12 semanas), consulta en el Servicio de Obstetricia para llevar a cabo estudios de control. Luego de la anamnesis y el examen físico general, se le realiza una ecografía, donde se observa un aumento de la translucencia nucal (TN) y pruebas serológicas (análisis de los marcadores séricos Proteína A del plasma sanguíneo asociada al embarazo (PAPP-A), y la subunidad beta libre de la gonadotropina coriónica humana (ß-hCG)) que muestran alteraciones compatibles con diagnóstico de Síndrome de Down. Frente a esta situación, la paciente consulta sobre la posibilidad de realizar algún otro estudio para determinar con mayor exactitud si su bebé presenta esta patología. Ante esta situación, el médico residente que acompaña al médico especialista, decide preguntarle si existe en esta etapa del embarazo algún otro método de screening no invasivo más sensible con el que se pueda determinar Síndrome de Down. Ya que recuerda que en una clase de obstetricia habían hablado de la detección de anomalías cromosómicas a partir de sangre materna, midiendo ADN fetal libre.

\section{Pregunta estructurada}

En mujeres cursando el primer trimestre de embarazo (pacientes), ¿es el estudio del ADN fetal circulante en comparación con el screening combinado (comparación) el método diagnóstico más sensible y específico con el que se puede detectar el Síndrome de Down (resultado)?

\section{Contexto clínico}

Los estudios prenatales son exámenes de suma importancia para controlar la salud de la madre y el feto durante el embarazo para detectar distintas afecciones y anomalías que no se podría hacer hasta el nacimiento. Las anormalidades cromosómicas son el resultado de alguna afección en uno de los 23 pares de cromosomas. El complemento cromosómico normal para el ser humano es 46, XY o 46, XX, según sea hombre o mujer respectivamente. El múltiplo exacto del número haploide de cromosomas (23) se denomina euploide. Las anomalías cromosómicas, o aneuploidías son alteraciones en los cromosomas sexuales o de los autosomas que pueden ser estructurales o numéricas. Existe una aberración numérica cuando el complemento cromosómico no es múltiplo de 23 , en este caso se habla de una aneuploidía. De esta manera podemos encontrar: monosomías (falta de un cromosoma), disomías (los cromosomas provienen de un mismo progenitor), trisomías (un cromosoma extra) y tetrasomías (2 o más cromosomas extras). Las alteraciones estructurales se pueden clasificar en: deleciones, inversiones, translocaciones, isocromosomas y cromosoma en anillo ${ }^{1}$.

El Síndrome de Down o trisomía 21 es una de las anomalías cromosómicas más frecuentes en nuestro medio, donde el $95 \%$ de las afecciones se deben a una trisomía completa. También puede originarse por una translocación robertsoniana entre cromosomas acrocéntricos o por mosaicos entre otras líneas celulares. Esta patología cursa

Revista Methodo: Investigación Aplicada a las Ciencias Biológicas. Facultad de Medicina. Universidad Católica de Córdoba. Jacinto Ríos 571 Bo Gral. Paz. X5004FXS. Córdoba. Argentina. Tel.: (54) 351 4517299 / Correo: methodo@ucc.edu.ar / Web: methodo.ucc.edu.ar| BIOESTADISTICA Y METODOLOGIA APLICADA Methodo 2019;4 (4):148-152. 
con un patrón característico de signos dismórficos, discapacidad intelectual y múltiples defectos congénitos asociados ${ }^{2}$.

Estas aneuploidías se asocian a alteraciones en el desarrollo que pueden conducir a retraso mental importante y/o defectos físicos y funcionales, lo que es una importante causa de morbimortalidad. Por esto, es importante la sospecha diagnóstica, en el cual se tienen en cuenta los siguientes factores de riesgo, como la edad materna igual o mayor a 35 años, antecedentes familiares de alteraciones cromosómicas, embarazos previos anormales y signos de alerta ecográficos. Hoy en día los métodos más utilizados para el screening prenatal de estas anomalías son los marcadores serológicos (la ß-hCG y la PAPPA) y las pruebas de imágenes ecográficas (el valor de la $\mathrm{TN}$ ). Aunque con el avance de las técnicas de diagnóstico prenatal, como el test de ADN fetal libre, se está buscando detectar de manera más sencilla y específica el síndrome de Down en el primer trimestre del embarazo ${ }^{3}$.

En las últimas décadas se ha intentado cambiar el paradigma para utilizar métodos de diagnósticos no invasivas en lugar de sólo métodos invasivos de diagnóstico prenatal (como la amniocentesis, pruebas de vellosidades coriales o la cordocentesis), ya que se encuentran asociados a un riesgo de aborto del $1 \%{ }^{3}$.Las pruebas de diagnóstico prenatal no invasivas, se basan en la detección ADN fetal que circula libremente en plasma materno; el cual puede ser analizado a partir de una muestra de sangre periférica de la madre desde el primer trimestre del embarazo. Utilizándose técnicas de PCR digital o tecnologías de secuenciación de nueva generación como métodos no invasivos para detectar alteraciones moleculares genéticas fetales a través de ADN fetal libre. Este método diagnóstico busca disminuir las indicaciones de pruebas diagnósticas invasivas utilizadas actualmente, como lo son la amniocentesis y la biopsia de vellosidades coriónicas; proponiendo una alternativa sin riesgo para la madre o el feto ${ }^{4}$.

\section{Búsqueda bibliográfica}

Para responder a la pregunta se realizó una búsqueda en PubMed utilizando el Mesh (Medical Subject Headings) "down syndrome AND genetics". El resultado inicial fue de 12048 artículos, tras lo cual se aplicaron los siguientes filtros: en relación al tipo de artículo buscado, systematic review y clinical trial; en cuanto a la fecha de publicación y población, los últimos 5 años y en humanos respectivamente. El número de resultados se redujo a 48 artículos. Tras la lectura de los títulos y resúmenes se seleccionó
"Cell free DNA Analysis for Noninvasive Examination of Trisomy", con un nivel de evidencia $1 \mathrm{~B}$.

\section{Resumen del artículo}

Este es un estudio prospectivo y multicéntrico, dónde participaron 35 centros internacionales. Estudiaron a mujeres embarazadas cursando entre la 10 y 14 semanas de gestación para el screening de aneuploidía utilizando el examen estándar y la detección de ADN fetal libre en sangre materna. El objetivo fue evaluar el riesgo de trisomía 21 en esta población. Además, se estudiaron otras aneuploidías como trisomía 13 y 18.

Para ello extrajeron sangre materna de 18,955 mujeres embarazadas de al menos 18 años de edad que se sometieron a un examen de aneuploidía durante el primer trimestre desde marzo de 2012 a abril 2013. El análisis primario incluyó 15.841 embarazadas, luego de aplicar criterios de exclusión, como el abandono en el seguimiento o errores en la recolección de las muestras, embarazos gemelares y embarazos por fertilización in vitro.

A las pacientes seleccionadas se le hicieron mediciones de la $\beta$-hCG, la PAPPA y la translucencia nucal. Estimaron el riesgo de aneuploidía utilizando un algoritmo que incluye los recuentos de ADN libre celular del cromosoma, la fracción fetal de ADN libre celular y el riesgo de trisomía a priori según la edad materna y gestacional. La determinación del resultado del nacimiento se basó en pruebas genéticas de diagnóstico, clasificándose a los recién nacidos como euploides o aneuploides.

El principal resultado fue el $\mathrm{ABC}$ de la curva ROC para la trisomía 21 con prueba de ADN fetal libre versus la detección estándar, también evaluaron el riesgo de trisomías 18 y 13. El ABC para la trisomía 21 fue de 0.999 para las pruebas de ADN fetal libre y de 0.958 para la detección estándar $(\mathrm{P}=0.001)$. La trisomía 21 se detectó en 38 de 38 mujeres (100\%; IC 95\%, 90,7 a 100) en el grupo que se estudió ADN fetal libre, en comparación con 30 de 38 mujeres (78,9\%; IC del 95\%, 62,7 a 90,4) en el grupo de detección estándar $(\mathrm{P}=0,008)$. Las tasas de resultados positivos falsos fueron de $0.06 \%$ (IC del $95 \%$, 0.03 a 0.11 ) en el grupo de ADN fetal libre y del $5.4 \%$ (IC del 95\%, 5.1 a 5.8) en el grupo de detección estándar ( $\mathrm{P}<0.001)$. El valor predictivo positivo para la prueba de ADN fetal libre fue del 80,9\% (IC del 95\%, 66,7 a 90,9), en comparación con el 3,4\% (IC del 95\%, 2,3 a 4,8) para la detección estándar $(\mathrm{P}<0,001)$. 
Otros de los resultados que obtuvieron fueron una edad materna promedio de 31 años y gestacional de 12.5 semanas. Hubo 68 anomalías cromosómicas: 38 fueron trisomía 21, 10 fueron trisomía 18, 6 fueron trisomía 13, 3 fueron 45, X, 3 fueron cromosomas marcadores, 2 fueron translocaciones desequilibradas, 2 fueron translocaciones balanceadas, y 1 fue cada una deleción 7p, eliminación / duplicación 5p, deleción 1q41 e isocromosoma Yp. La trisomía 21 se identificó en 38 de 15,841 mujeres, con una prevalencia de 1 en 417.

En el estudio analizado concluyen que las pruebas de ADN fetal libre para la trisomía 21 tuvieron mayor sensibilidad, menor tasa de falsos positivos y mayor valor predictivo positivo que la detección estándar con la medición de la translucidez nucal y los analitos bioquímicos. Análisis del artículo

En base al artículo seleccionado se tuvieron en cuenta diferentes variables estadísticas para comparar la validez de ambos métodos diagnósticos, y así poder responder a nuestra pregunta clínica estructurada. El diseño del trabajo es específico para un estudio de diagnóstico, por lo que se pudo inferir el valor diagnóstico para cada método. Dado que todas las embarazadas fueron sometidas a ambos estudios (screening standard y ADN fetal libre), y el resultado del nacimiento se basó en pruebas genéticas de diagnóstico clasificándose a los recién nacidos como euploides o aneuploides, puede concluirse que no se ha incurrido en sesgos de verificación, aunque pueden existir sesgos de expectativa dado que no se menciona que los médicos hayan sido cegados a los datos clínicos. Tampoco se menciona la calificación y número de los médicos participantes, ni si se calculó la concordancia intra e interobservador, hecho este que potencialmente podría aumentar indebidamente la variabilidad de los resultados observados.

Para el análisis se procedió definir los valores de la sensibilidad, la especificidad, el valor predictivo positivo y el valor predictivo negativo, con sus respectivos IC 95\%. Los mismos se calcularon tanto para el screening standard como para la prueba de ADN fetal libre, teniendo en cuenta los valores que a continuación se detallan (Tabla 1 y 2).
Tabla 1: Resultados de pacientes estudiados con el screening standard.

\begin{tabular}{|l|c|c|c|}
\hline & $\begin{array}{c}\text { Con Sindrome de } \\
\text { Down (+) }\end{array}$ & $\begin{array}{c}\text { Sin Sindrome } \\
\text { de Down (-) }\end{array}$ & Total \\
\hline $\begin{array}{l}\text { Screening } \\
\text { Standard (+) }\end{array}$ & 30 & 854 & 884 \\
\hline $\begin{array}{l}\text { Screening } \\
\text { Standard (-) }\end{array}$ & 8 & 14949 & 14957 \\
\hline Total & 38 & 15803 & 15841 \\
\hline
\end{tabular}

Tabla 2: Resultados de pacientes estudiados con la prueba de ADN fetal libre.

\begin{tabular}{|l|c|c|c|}
\hline & $\begin{array}{c}\text { Con sindrome } \\
\text { de Down (+) }\end{array}$ & $\begin{array}{c}\text { Sin Sindrome } \\
\text { de Down (-) }\end{array}$ & Total \\
\hline ADN fetal libre (+) & 38 & 9 & 47 \\
\hline ADN fetal libre (-) & 0 & 15794 & 15794 \\
\hline Total & 38 & 15803 & 15841 \\
\hline
\end{tabular}

Los resultados que se obtuvieron fueron una sensibilidad del 78,9\% (IC 95\%: 63,5 - 88,9) y especificidad del 94,59\% (IC 95\%: 94,2 - 94,9) para el screening standard; en cambio, el screening con ADN fetal libre presenta una sensibilidad del 100\% (IC 95\%: 90,8 - 100) y especificidad del 99,9\% (IC 95\%: 99,8 - 99,9). En cuanto a los resultados del VPP del método ecográfico y estudios serolgicos fue del 3,39\% (IC 95\%: 2,38 - 4,8), en comparación con 80,8\% (IC 95\%: 67,4 - 89,5) del método por ADN fetal libre. Con respecto al VPN fue de $99,9 \%$ (IC 95\%: 99,8 - 99,9) para el screening standard y de un 100\% (IC 95\%: 99,98 - 100) para el otro estudio.

Por otro lado, solo destacaremos el resultado del área bajo la curva de las curvas ROC, la cual relaciona la sensibilidad y especificidad de un método de estudio y otro. Lo que da una idea de hasta qué punto cada prueba diagnóstica es capaz de detectar los casos positivos correctamente entre todos los casos estudiados, es así que el ABC para el screening standard fue del 0,958, en cambio el ABC para el estudio del ADN fetal libre fue del $0,999(\mathrm{p}=0,001)$.

Revista Methodo: Investigación Aplicada a las Ciencias Biológicas. Facultad de Medicina. Universidad Católica de Córdoba. Jacinto Ríos 571 Bo Gral. Paz. X5004FXS. Córdoba. Argentina. Tel.: (54) 351 4517299 / Correo: methodo@ucc.edu.ar / Web: methodo.ucc.edu.ar| BIOESTADISTICA Y METODOLOGIA APLICADA Methodo 2019;4 (4):148-152. 


\section{Comentarios y recomendaciones finales}

Con la evidencia actual, se pudo demostrar que el screening con ADN fetal libre presenta una mayor sensibilidad y especificidad frente al screening combinado para el diagnóstico del Síndrome de Down. Por lo tanto, el estudio del ADN fetal sería una opción de estudio no invasivo para el diagnóstico de anomalías cromosómicas. A pesar de lo expuesto anteriormente, no podemos evitar tener en cuenta la gran limitación que supone el elevado costo del screening de ADN fetal libre para poder estandarizar su uso.

\section{Bibliografía}

1. Kumar V, Abbas A, Aster J. Robbins y Cotran. Trastornos Genéticos. En: Robbins y Cotran. Patología estructural y funcional. $9^{\mathrm{a}}$ ed. Barcelona: Elsevier. 2015. p.137 -142.

2. Rubio González T; Norbert Vázquez L; García González D. Evaluation of growth and development of patients with Down syndrome in Santiago de Cuba. MediSan. 2018; 22 (01): 19-26.

3. Nicolaides K. H. Screening for chromosomal defects.Ultrasound Obstet Gynecol. 2003; 21 (4): 313-21.

4. Breveglieri G, D'Aversa E, Finotti A, Borgatti M. Non-invasive Prenatal Testing
Using Fetal DNA. Mol Diagn Ther. 2019; 23 (2): 291-299.

5. Norton ME, Jacobsson B, Swamy GK, Laurent LC, et al. Cell-free DNA analysis for noninvasive examination of trisomy. $\mathrm{N}$ Engl J Med 2015; 372:1589-97.

Link:

https://www.youtube.com/watch?v=kjdGxZwT $\mathrm{vSw} \& \mathrm{t}=632 \mathrm{~s}$ transmisión del ateneo central Facultad Ciencias de la Salud.

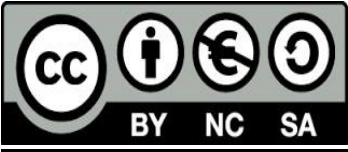

Revista Methodo: Investigación Aplicada a las Ciencias Biológicas. Facultad de Medicina. Universidad Católica de Córdoba. Jacinto Ríos 571 Bo Gral. Paz. X5004FXS. Córdoba. Argentina. Tel.: (54) 351 4517299 / Correo: methodo@ucc.edu.ar / Web: methodo.ucc.edu.ar| BIOESTADISTICA Y METODOLOGIA APLICADA Methodo 2019;4 (4):148-152. 